\title{
PRP, PRF E PLASMA GEL PARA DISFUNÇÕES ESTÉTICAS
}

\author{
PRP, PRF AND PLASMA GEL FOR AESTHETIC DYSFUNCTIONS
}

\author{
Daniela Lopez ${ }^{1}$ \\ Fabiano de Abreu Rodrigues ${ }^{2}$
}

RESUMO: Plasma é um Líquido tecidular, sendo considerado $55 \%$ do sangue, além disso, 92\% constituído por água, utilizado como solvente em $\mathrm{pH} 7.4$, contém substâncias de diversos pesos moleculares que perfazem $7 \%$ do seu volume. O Plasma Rico em Plaquetas (PRP) é um produto derivado do sangue autólogo, que em seu preparo possui o objetivo de um produto com alta concentração de plaquetas em um pequeno volume de plasma. O Plasma Rico em fibrina (PRF), é um produto com alta concentração de plaquetas em uma única membrana de fibrina, com todos os componentes de uma amostra sanguínea favoráveis para a reparação tecidual. Sendo assim, o objetivo foi Compreender as diferentes formas do plasma, em PRP, PRF e em gel e entender suas funções e benefícios para os procedimentos estéticos. Trata-se de uma revisão de literatura no portal de periódicos eletrônicos da SciELO, PubMed e Science Direct. Em todos os estudos encontrados nesta revisão, se apresentaram benéficos ao rejuevenscimento, sendo confirmados tais resultados por meio de diferentes análises.

Palavras- chaves: Plasma rico em plaquetas. Plasma. Plasma gel. Plasma rico em fibrina.

\section{INTRODUÇÃO}

\section{I.I Plasma}

É um líquido tecidular, sendo considerado $55 \%$ do sangue, além disso, 92\% constituído por água, utilizado como solvente em $\mathrm{pH}$ 7.4, contém substâncias de diversos pesos moleculares que perfazem 7\% do seu volume (Feigin, 2019).

\footnotetext{
${ }^{I}$ Graduada em estética e cosmetologia,Pós- graduada em procedimentos intradérmicos e subcutâneos, Especialista em Cosmiatria Orgânica e Ortomolecular, Presidente da sociedade brasileira de estética e cosmetologia, Pós graduada em biomedicina estética, Pós graduanda em Estética e Cosmetologia Avançada UNIFESP - Universidade Federal de São Paulo, Residência em Intradermoterapia Estética T.A.E Pesquisadora em cosmetologia em prol do Envelhecimento, curso integrado a UNICAMP. Email: daniela.lopezz@hotmail.com.

${ }^{2} \mathrm{PhD}$, neurocientista, mestre psicanalista, biólogo, historiador, antropólogo, com formações também em neuropsicologia, psicologia, neurolinguística, neuroplasticidade, inteligência artificial, neurociência aplicada à aprendizagem, filosofia, jornalismo e formação profissional em nutrição clínica - Diretor do Centro de Pesquisas e Análises Heráclito; Chefe do Departamento de Ciências e Tecnologia da Logos University International, UniLogos; Membro da Federação Européia de Neurociências e da Sociedade Brasileira e Portuguesa de Neurociências. Universidades em destaque: Logos University International, UniLogos, Nova de Lisboa, Faveni, edX Harvard, Universidad de Madrid.
} 
Tais componentes são em sua maioria, proteínas plasmáticas, nutrientes, gases respiratório, hormônios dentre outros (Feigin, 2019).

Em relação as proteínas plasmáticas, a que está presente em maior quantidade no plasma é a albumina, que possui funções de regulação da pressão osmótica do sangue, as imunoglobulinas $G$ com papel ativo no sistema imunitário, fibrinogénio e fatores de coagulação que possui um ação crucial na hemóstase (Plebani, 2020).

O plasma pode ser utilizado em transfusões para fins terapêuticos, médicos ou estéticos, porém já existem produtos farmacêuticos derivados do plasma (Plebani, 2020).

\subsection{Plasma Rico em Plaquetas (PRP)}

Produto derivado do sangue autólogo, que em seu preparado possui o objetivo de um produto com alta concentração de plaquetas em um pequeno volume de plasma (Everts, 2020).

O uso do PRP teve início na década de 9o, para os tratamentos odontológicos, como: cirurgia oral, reconstrutiva oral, bucomaxilofacial e procedimentos de reconstrução para implantodontia, bem como nos demais tratamentos de saúde, reparação tecidual e a regeneração óssea (Everts, 2020).

Sua aplicação tem o objetivo de acelecerar o processo de hemostasia, cicatrização de feridas e reepitelização. Por meio dos PRP tambem é liberado os fatores de crescimento, para estímulo da angiogênese, mitose celular, quimiotaxia dos neutrófilos, macrófagos e fibroblastos (Everts, 2020).

\subsection{Plasma Rico em Fibrina (PRF)}

Desenvolvido na França, pelo médico e pesquisador Joseph Choukroun, com o objetivo do uso específico em cirurgia buco-maxilo-facial. O PRF, é um produto com alta concentração de plaquetas em uma única membrana de fibrina, com todos os componentes de uma amostra sanguínea favoráveis para a reparação tecidual (Simonpieri, 2012).

Para obtenção da PRF, é necessária uma amostra de sangue, sem anticoagulante, em tubos de roml que são centrifugados a 3000 
rpm(aproximadamente 40og) por Io minutos. Para esta técnica é necessária somente a centrifugação do sangue sem adicionais (Simonpieri, 2012).

A L-PRF, promove o aumento da fixação dos osteoblastos, bem como sua proliferação, e em conjunto regula a produção de proteína colágeno (Simonpieri, 2012).

\subsection{Plasma Gel}

A formação do plasma gel, é realizada a partir das alterações do plasma a partir da mudança de sua textura, através da temperatura, $\mathrm{pH}$ e concentração de proteínas, tentando manter as características de $\mathrm{pH}$ e interação entre as proteínas, o aquecimento com variação de $70^{\circ} \mathrm{C}$ a $90^{\circ} \mathrm{C}$ (Piccin, 2017).

O plasma é separado em uma seringa de $5 \mathrm{~mL}$, realizam-se incubações seriadas de $37^{\circ}, 70^{\circ}$ e $90^{\circ} \mathrm{C}$ para desnaturação e geleificação, obtendo resultado em $\mathrm{mm} 2 / \mathrm{g}$ (Piccin, 2017).

\section{OBJETIVO}

Compreender as diferentes formas do plasma, em PRP, PRF e em gel e entender suas funções e benefícios para os procedimentos estéticos.

\section{METODOLOGIA}

Trata-se de uma revisão de literatura no portal de periódicos eletrônicos da SciELO, PubMed e Science Direct. Sendo utilizado os seguintes termos para realização da busca de dados em português: plasma rico em plaquetas, plasma, plasma gel, plasma rico em fibrina e em inglês: platelet rich plasma, plasma, plasma gel, fibrin rich plasma.

\section{RESULTADOS E DISCUSSÃO}

A revisão foi construída por 6 estudos entre eles, séries de casos e ensaios clínicos randomizados, com indivíduos de ambos com mulheres de diferentes idades e diferentes métodos de avaliação. Dois destes estudos avaliaram o plasma rico em plaquetas, outros dois, plasma rico em fibrinas e o restante, plasma gel. 


\begin{tabular}{|c|c|c|c|c|}
\hline $\begin{array}{l}\text { Autor } \\
\text { (Ano) }\end{array}$ & Tipo de estudo & Amostra & Produto & Análises \\
\hline $\begin{array}{l}\text { Alam et. } \\
\text { al. (2018) }\end{array}$ & $\begin{array}{l}\text { Ensaio clínico } \\
\text { randomizado }\end{array}$ & 27 mulheres & $\begin{array}{c}\text { Plasma rico em plaquetas } \\
\text { para o rejuvenescimento } \\
\text { da pele facial } \\
\text { fotoenvelhecida }\end{array}$ & $\begin{array}{c}\text { Pontuação de } \\
\text { envelhecimento e } \\
\text { autoavaliação }\end{array}$ \\
\hline $\begin{array}{l}\text { Everts et. } \\
\text { al. (2019) }\end{array}$ & Série de casos & II mulheres & $\begin{array}{l}\text { Plasma rico em plaquetas } \\
\text { e pobre em neutrófilos }\end{array}$ & $\begin{array}{l}\text { Volume das rugas, } \\
\text { firmeza da pele, } \\
\text { autoavaliação }\end{array}$ \\
\hline $\begin{array}{l}\text { Hassam } \\
\text { et. al. } \\
(2020)\end{array}$ & Série de casos & II mulheres & Plasma rico em fibrinas & $\begin{array}{l}\text { Poros, manchas } \\
\text { escuras, }\end{array}$ \\
\hline $\begin{array}{l}\text { Hu et. al. } \\
\quad(202 \mathrm{I})\end{array}$ & $\begin{array}{l}\text { Ensaio clínico } \\
\text { randomizado }\end{array}$ & 30 mulheres & Plasma rico em fibrinas & $\begin{array}{l}\text { Textura da pele, } \\
\text { rugas }\end{array}$ \\
\hline $\begin{array}{l}\text { Neinaa et. } \\
\text { al. (2020) }\end{array}$ & $\begin{array}{l}\text { Ensaio clínico } \\
\text { randomizado }\end{array}$ & 68 mulheres & Plasma gel & $\begin{array}{c}\text { Grau de } \\
\text { hiperpigmentação e } \\
\text { da escala de } \\
\text { classificação do } \\
\text { vale lacrimal para } \\
\text { avaliar olheiras }\end{array}$ \\
\hline $\begin{array}{l}\text { Doghaim } \\
\text { et. al. } \\
\text { (2019) }\end{array}$ & Série de casos & 52 mulheres & Plasma gel & $\begin{array}{l}\text { Textura da pele e } \\
\text { fotografia }\end{array}$ \\
\hline
\end{tabular}

Fonte: Autor

No estudo de Alam et. al. 2018, realizado com indivíduos entre 18 a 70 anos, que apresentassem nível II ou superior na escala de glogau, a intervenção foi realizada nas bochecha bilaterais, com injeções intradérmicas de $3 \mathrm{~mL}$ de PRP e do lado controle solução salina normal estéril. Nenhum dos indivíduos obteve nenhum efeito adverso.

Apresentando diferença significativa entre PRP e solução salina normal para linhas finas. Após seis meses os participantes, autoavaliaram e relataram satisfação no tratamento (Alam et. al. 2018).

Em outro estudo, onde onze voluntários, foram tratados com 3 sessões de PRP. Apresentando diminuição significativa na contagem e área de manchas marrons, após 3 meses de tratamento. Em relação a contagem e o volume das rugas 
foram significativamente reduzidos. Os parâmetros de firmeza da pele melhoraram significativamente, a vermelhidão da pele melhorou significativamente após I69 dias após a terapia para ambas as áreas nasolabial e malar. (Everts et. al. 2019)

Hassam et. al. 2020, avaliou o rejuvenescimento a partir de 3 injeções de plasma rico em fibrinas por um intervalo de 3 em 3 meses. Demonstrando melhora significativa nas manchas da superfície e poros, durante o acompanhamento de 3 meses. Em relação a satisfação das voluntárias medida por meio da escala FACE-Q mostraram uma melhora significativa desde o início, incluindo satisfação com a pele.

Outro estudo para avaliar os efeitos do plasma rico em fibrinas, foi realizado por meio de um ensaio clínico randomizado, o grupo controle constituído por is voluntárias e 14 tratados, durante seis semanas, apresentando melhora na textura e rugas da pele dos indivíduos tratados em comparação ao grupo controle (Hu et. al. 202I).

Com o objetivo de avaliar os efeitos do plasma gel, para o rejuvenescimento infraorbital, sendo controle o lado não tratado e o lado intervenção tratado, foram avaliados após três sessões com intervalo de 2 semanas e acompanhados mensalmente por 3 meses. O lado tratado obteve redução significativa do grau de hiperpigmentação e da escala de classificação do vale lacrimal. (Neinaa et. al. 2020).

Outro estudo para avaliar da ação do plasma gel, foi realizado com um intervalo de 2 semanas e acompanhados mensalmente por 3 meses. Apresentando melhora significativa imediata após as injeções, se mostrando mantidos até o período final de avaliação. Os níveis de textura da pele também foram benéficos. A técnica não demonstrou efeitos colaterais. (Doghaim et. al. 2019)

\section{CONSIDERAÇÕES FINAIS}

Por meio dos resultados apresentados acima, dos ensaios clínicos para avaliação da ação do rejuvenescimento estético, a partir dos tratamentos e protocolos propostos com plasma rico em plaquetas, plasma rico em fibrina ou plasma em gel, todos estes estudos, encontrados nesta revisão, se apresentaram benéficos ao rejuevenscimento, sendo confirmados tais resultados por meio de diferentes análises. 


\section{REFERÊNCIA}

ALAM, M., Hughart, R., Champlain, A., Geisler, A., Paghdal, K., Whiting, D., Hammel, J.A., Maisel, A., Rapcan, M.J., West, D.P., Poon, E. Effect of Platelet-Rich Plasma Injection for Rejuvenation of Photoaged Facial Skin: A Randomized Clinical Trial. JAMA Dermatol. n. 154, v. 12, págs. I447-1452, 2018 doi: I0.IOoI/jamadermatol.2018.3977.

DOGHAIM, N.N., El-Tatawy, R.A., Neinaa, Y.M.E. Assessment of the efficacy and safety of platelet poor plasma gel as autologous dermal filler for facial rejuvenation. J Cosmet Dermatol, 2019 doi: 10.11I/jocd.12876.

EVERTS, P., Onishi, K., Jayaram, P., Lana, J.F., Mautner, K. Platelet-Rich Plasma: New Performance Understandings and Therapeutic Considerations in 2020. Int J Mol Sci. n. 2I, v. 20, págs. 7794, 2020 doi: 10.3390/ijms21207794.

EVERTS, P.A., Pinto, P.C., Girão, L. Autologous pure platelet-rich plasma injections for facial skin rejuvenation: Biometric instrumental evaluations and patient-reported outcomes to support antiaging effects. J Cosmet Dermatol. n. I8, v. 4, págs. 985-995, 2019 doi: 10.1111/jocd.12802.

FEIGIN, K., Shope, B. Use of Platelet-Rich Plasma and Platelet-Rich Fibrin in Dentistry and Oral Surgery: Introduction and Review of the Literature. J Vet Dent. n. 36, v. 2, págs. 109-123, 2019 doi: 10.1177/o898756419876057

HASSAN, H., Quinlan, D.J., Ghanem, A. Injectable platelet-rich fibrin for facial rejuvenation: A prospective, single-center study. J Cosmet Dermatol. n. I9, v. I2, págs. 3213-322, 2020 doi: 10.11II/jocd.13692.

PICCIN, A., Di Pierro, A.M., Canzian, L., Primerano, M., Corvetta, D., Negri, G., Mazzoleni, G., Gastl, G., Steurer, M., Gentilini, I., Eisendle, K., Fontanella, F. Platelet gel: a new therapeutic tool with great potential. Blood Transfus. n.15, v. 4, págs. 333-340, 2017 doi: 10.2450/2016.0038-16.

PlebANI, M., Banfi, G., Bernardini, S., Bondanini, F., Conti, L., Dorizzi, R., Ferrara, F.E., Mancini, R., Trenti, T. Serum or plasma? An old question looking for new answers. Clin Chem Lab Med. n. 28, v. 2, págs. 178-187, 2020 doi: $10.1515 / \mathrm{cclm}$ 2019-0719.

SIMONPIERI, A,, Del Corsom, M., Vervelle, A., Jimbo, R., Inchingolo, F., Sammartino, G., Dohan, Ehrenfest, D.M. Current knowledge and perspectives for the use of platelet-rich plasma (PRP) and platelet-rich fibrin (PRF) in oral and maxillofacial surgery part 2: Bone graft, implant and reconstructive surgery. Curr Pharm Biotechnol. n. 13, v. 7, págs. 1231-56, 2012 doi: 10.2174/138920112800624472. 